\title{
A spectral operator-theoretic framework for global stability
}

\author{
Alexandre Mauroy and Igor Mezić
}

\begin{abstract}
The global description of a nonlinear system through the linear Koopman operator leads to an efficient approach to global stability analysis. In the context of stability analysis, not much attention has been paid to the use of spectral properties of the operator. This paper provides new results on the relationship between the global stability properties of the system and the spectral properties of the Koopman operator. In particular, the results show that specific eigenfunctions capture the system stability and can be used to recover known notions of classical stability theory (e.g. Lyapunov functions, contracting metrics). Finally, a numerical method is proposed for the global stability analysis of a fixed point and is illustrated with several examples.
\end{abstract}

\section{INTRODUCTION}

The operator-theoretic approach to dynamical systems provides a powerful insight into stability theory. As an illustration, the duality between Lyapunov functions and densities, discovered only some years ago in [14], is directly derived from the duality, known for decades, between two adjoint operators describing the system-namely, the Koopman operator and the Perron-Frobenius operator. Also, the operator-theoretic approach considers the system as a whole, since one iteration of the (infinite-dimensional) operator is equivalent to an iteration along all the system trajectories. The framework is therefore well-suited to global stability analysis.

The development of an operator-theoretic framework for stability analysis is still in its infancy. The main contributions can be found in [13], [15] and focus almost exclusively on the Perron-Frobenius operator. Since the (Koopman and PerronFrobenius) operators are linear, it is also natural to consider their spectral properties. However, while these properties were successfully used to capture the system behavior on the attractor (see e.g. [8], [10]), they have never been exploited in the context of stability theory.

In this paper, we set the basis of a novel stability theory based on spectral operator-theoretic methods. In particular, we focus on the spectral properties of the Koopman operator and we establish a relationship between global stability and the existence of specific eigenfunctions. Beyond their relationship to stability, the eigenfunctions are related to important geometric properties of the dynamics (derivation of special Lyapunov functions and contracting metrics, global linearization [6], isostables [9], etc.). This analysis mirrors the spectral approach to stability in linear systems.

A. Mauroy and I. Mezić are with the Department of Mechanical Engineering, University of California, Santa Barbara, CA 93106-5070 USA. alex.mauroy@engr.ucsb.edu, mezicdengr.ucsb.edu
Although most of the results are general and make no assumption on the nature of the attractor, we consider with more details the case of stable fixed points. In this situation, we propose a numerical method for computing smooth eigenfunctions. Without computing the system trajectories, this method is used to investigate the stability of the fixed point and to estimate the size of its basin of attraction.

The paper is organized as follows. In Section II, we introduce the Koopman operator framework and briefly discuss the duality with the Perron-Frobenius operator. The relationship between classical Lyapunov stability and the properties of the Koopman operator is presented in Section III. Section IV develops our main results, deriving global stability results from the spectral properties of the operator. The numerical method is developed in Section V and is used to investigate the stability properties of several dynamical systems with globally stable fixed points. Finally, we give some concluding remarks in Section VI.

\section{AN OPERATOR-THEORETIC FRAMEWORK}

In this section, we give an overview of the existing operator-theoretic framework for the analysis of the dynamical system

$$
\dot{x}=F(x), \quad x \in \mathbb{R}^{n} .
$$

We denote by $\varphi(t, x): \mathbb{R} \times \mathbb{R}^{n} \rightarrow \mathbb{R}^{n}$, or equivalently $\varphi^{t}(x)$, the flow induced by the system, i.e. $\varphi^{t}(x)$ is the solution of (1) associated with the initial condition $x \in \mathbb{R}^{n}$. Unless otherwise specified, we only require existence and uniqueness of the flow $\varphi^{t}$. (The vector field $F$ is assumed to be Lipschitz continuous, but could also be replaced by a well-defined hybrid system.)

\section{A. The Koopman operator}

Consider a compact set $X \subset \mathbb{R}^{n}$ which is forward invariant under $\varphi^{t}(\cdot)$. (Note that many of the following results also hold when $X=\mathbb{R}^{n}$ or when $X$ is a general compact metric space.) The Koopman operator describes the evolution of observables $f: X \rightarrow \mathbb{C}$ along the trajectories of (1).

Definition 1 (Koopman operator): For a given space $\mathcal{F}$ of observables, the Koopman (semi)group of operators $U^{t}$ : $\mathcal{F} \rightarrow \mathcal{F}$ associated with (1) is defined by

$$
U^{t} f=f \circ \varphi^{t} \text {. }
$$

While the system (1) may be nonlinear, the Koopman operator (2) is always linear. If $f$ and $F$ are continuously differentiable, $f(t, x)=U^{t} f_{0}(x)$ satisfies the partial differential equation (see e.g. [7])

$$
\frac{\partial f}{\partial t}=F \cdot \nabla f \triangleq L_{U} f
$$


with the initial condition $f(0, x)=f_{0}(x)$. The symbol $\nabla$ denotes the gradient and $\cdot$ is the inner product in $\mathbb{R}^{n}$. The operator $L_{U}$ is the infinitesimal generator of $U^{t}$, i.e. $L_{U} f=\lim _{t \rightarrow 0}\left(U^{t} f-f\right) / t$. Note that no boundary condition is needed with (3) since $X$ is forward invariant.

At this point, we can already point out a relationship between the Koopman operator and stability theory. If (1) admits a stable fixed point $x^{*} \in X$, a Lyapunov function for the system is interpreted as a (nonnegative) observable that satisfies $L_{U}^{t} \mathcal{V}(x)<0 \forall x \neq x^{*}$. It is therefore clear that stability is closely related to the properties of the Koopman operator.

\section{B. Remark on duality}

The Koopman operator has a counterpart which describes the transport of densities $\rho: X \rightarrow \mathbb{C}$ along the trajectories of (1). This dual operator is the Perron-Frobenius operator $P^{t}: \mathcal{F}^{\dagger} \rightarrow \mathcal{F}^{\dagger}$, which can be defined as the adjoint of the Koopman operator, that is

$$
\left\langle U^{t} f, \rho\right\rangle=\left\langle f, P^{t} \rho\right\rangle, \quad \forall f \in \mathcal{F}, \forall \rho \in \mathcal{F}^{\dagger}
$$

with the inner product

$$
\langle f, \rho\rangle=\int_{X} f(x) \rho^{c}(x) d x,
$$

where $\rho^{c}$ denotes the complex conjugate of $\rho$.

If $\rho$ and $F$ are continuously differentiable, one can show that $\rho(t, x)=P^{t} \rho_{0}(x)$ satisfies the transport equation (see e.g. [7])

$$
\frac{\partial \rho}{\partial t}=-\nabla \cdot(F \rho) \triangleq L_{P} \rho
$$

with the initial condition $\rho(0, x)=\rho_{0}(x)$.

The Koopman and Perron-Frobenius operators provide two dual descriptions of the system (i.e. point-wise or set-wise description). Also, they naturally yield two dual methods for stability analysis. In the case of a fixed point $x^{*}$, while a Lyapunov function decreases under the action of $U^{t}$, a Lyapunov density (or Lyapunov measure) decreases (almost everywhere) under the action of $P^{t}$ [15]. The Lyapunov density was initially introduced in [14] as a function $C^{1}\left(X \backslash\left\{x^{*}\right\}\right)$ that satisfies $\nabla \cdot(F \rho)>0$, a property which precisely corresponds to the action of the Perron-Frobenius infinitesimal generator $L_{P} \rho<0$, according to (4) (see also [13]).

The main interest of the Perron-Frobenius approach is that it leads to the weaker notion of almost everywhere stability (in contrast to classical stability). However, the Koopman approach is more suited to the spectral method that we propose here. In the remaining of the paper, we therefore focus on the Koopman operator framework.

\section{RELATIONSHIP TO STABILITY}

We assume that (1) admits an attractor $A \subset X$ and we study the stability of $A$ through the Koopman operator framework. The results are general since they make no assumption on the type of attractor. For instance, $A$ can even be a set of attractors (e.g. several stable fixed points).
Consider the subspace $\mathcal{F}_{A_{c}} \subseteq \mathcal{F}$ of functions with support on $A_{c}=X \backslash A$, i.e.

$$
\mathcal{F}_{A_{c}}=\{f \in \mathcal{F} \mid f(x)=0 \forall x \in A\} .
$$

For $x \in A$, one has $\varphi^{t}(x) \in A$ for all $t \in \mathbb{R}$, so that $U^{t} f=$ $f \circ \varphi^{t}(x)=0$ if $f \in \mathcal{F}_{A_{c}}$. It follows that $\mathcal{F}_{A_{c}}$ is invariant under $U^{t}$, and we denote by $U_{A_{c}}^{t}$ the restriction of $U^{t}$ to $\mathcal{F}_{A_{c}}$. Similarly, one can also consider the Koopman operator $U_{A}^{t}$ which is related to the flow on the attractor and which acts on observables restricted to $A$, i.e. $\left.f\right|_{A}: A \rightarrow \mathbb{C}$. The operator $U_{A}^{t}$ is rigorously defined by $U_{A}^{t}\left(\left.f\right|_{A}\right)=\left.\left(U^{t} f\right)\right|_{A}$.

The stability of (1) is directly related to the properties of the restriction $U_{A_{c}}^{t}$.

Proposition 1 (Transience): The attractor $A$ of (1) is globally asymptotically stable in $X$, i.e. the limit set of every trajectory is contained in $A$, if and only if

$$
\lim _{t \rightarrow \infty} U_{A_{c}}^{t} f=0 \quad \forall f \in \mathcal{F}_{A_{c}},
$$

with $\mathcal{F}=C^{0}(X)$.

Proof: Sufficiency. Consider the distance function

$$
f(x)=\min _{y \in A}\|x-y\|, \quad x \in X .
$$

The function is continuous and has zero value on $A$. Then, it follows from (5) that $A$ is globally asymptotically stable. Necessity. For $f \in \mathcal{F}_{A_{c}}$, we have

$$
\lim _{t \rightarrow \infty} U_{A_{c}}^{t} f(x)=\lim _{t \rightarrow \infty} f \circ \varphi^{t}(x)=f \circ \lim _{t \rightarrow \infty} \varphi^{t}(x),
$$

since $f$ is continuous. The limit set of $\varphi^{t}(x)$ is in $A$, so that one gets $\lim _{t \rightarrow \infty} U_{A_{c}}^{t} f(x)=0$ for all $x \in X$.

In the case of exponential stability, the following proposition shows a similar relationship to the properties of the Koopman operator, in the space $\operatorname{Lip}(X)$ of Lipschitz continuous functions.

Proposition 2 (Geometric decay): Consider the map $\pi$ : $X \rightarrow A$ defined by

$$
\pi(x)=\arg \min _{y \in A}\|x-y\| .
$$

The attractor is exponentially stable in $X$, i.e. there exist positive constants $C$ and $\sigma$ such that

$$
\left\|\varphi^{t}(x)-\pi\left(\varphi^{t}(x)\right)\right\| \leq C e^{-\sigma t}\|x-\pi(x)\| \quad \forall x \in X,
$$

if and only if there exist positive constants $C$ and $\sigma$ such that

$$
\left|U_{A_{c}}^{t} f\right| \leq C \frac{K_{1}}{K_{2}} e^{-\sigma t}|f| \quad \forall f \in \mathcal{F}_{A_{c}},
$$

for all $\mathcal{F}=\{f \in \operatorname{Lip}(X)|\forall x, y \in X| f,(x)-f(y) \mid \leq$ $\left.K_{1}\|x-y\|,|f(x)-f(\pi(x))| \geq K_{2}\|x-\pi(x)\|, K_{1}, K_{2}>0\right\}$.

Proof: Sufficiency. The distance function

$$
f(x)=\|x-\pi(x)\|=\min _{y \in A}\|x-y\|, \quad x \in X
$$

is a Lipschitz continuous function of $\mathcal{F}_{A_{c}}$ with $K_{1}=K_{2}=$ 1 . Then, it follows from (6) that $A$ is exponentially stable. 
Necessity. If $f \in \mathcal{F}_{A_{c}}$, exponential stability implies

$$
\begin{aligned}
\left|U_{A_{c}}^{t} f(x)\right| & =\left|U_{A_{c}}^{t} f(x)-U_{A_{c}}^{t} f(\pi(x))\right| \\
& \leq K_{1}\left\|\varphi^{t}(x)-\pi\left(\varphi^{t}(x)\right)\right\| \\
& \leq C K_{1} e^{-\sigma t}\|x-\pi(x)\| \\
& \leq C \frac{K_{1}}{K_{2}} e^{-\sigma t}|f(x)-f(\pi(x))| \\
& =C \frac{K_{1}}{K_{2}} e^{-\sigma t}|f(x)|
\end{aligned}
$$

where we used $f(\pi(x))=0$.

Remark 1 (Lyapunov function): When $A$ is a fixed point $x^{*}$, the existence of a Lyapunov function $\mathcal{V} \in \mathcal{F}_{A_{c}}$ directly implies (5) $\forall f \in C^{1}(X)$. Indeed, for every $f \in$ $\mathcal{F}_{A_{c}} \cap C^{1}(X)$, there exists a constant $C$ such that $f(x) \leq$ $C \mathcal{V}(x) \forall x \in X$. Therefore, one has $\lim _{t \rightarrow \infty} f\left(\varphi^{t}(x)\right) \leq$ $C \lim _{t \rightarrow \infty} \mathcal{V}\left(\varphi^{t}(x)\right)=0$ for all $x \in X$. Also, when the fixed point is exponentially stable, a Lyapunov function is given by

$$
\mathcal{V}(x)=\int_{0}^{\infty} U_{A_{c}}^{\tau} f(x) d \tau
$$

If $f \in \mathcal{F}_{A_{c}}$ is a nonnegative function of the space $\mathcal{F}$ considered in Proposition 2 (e.g. $f(x)=\left\|x-x^{*}\right\|$ ), it follows from (6) that (7) is integrable. Then, one easily verifies that

$$
\frac{d}{d t} \mathcal{V}\left(\varphi^{t}(x)\right)=-\int_{0}^{\infty} \frac{d}{d t} f\left(\varphi^{t+\tau}(x)\right) d \tau=-f\left(\varphi^{t}(x)\right)<0 .
$$

(Note that we have $\mathcal{V}=-L_{U}^{-1} f$.) This result can be seen as the continuous equivalent of a converse Lyapunov theorem proved in [15].

\section{SPECTRAL ANALYSIS}

The results described in the previous section are difficult to use in practice for the stability analysis of the system. However, since the Koopman operator is linear, it is natural to exploit its spectral properties. In this section, we show that these spectral properties are related to the stability of the system.

Studying the point spectrum alone is sufficient for our purpose, so that the continuous and residual parts of the spectrum are not considered here. They are either empty with most of the types of attractors (fixed point, limit cycle, quasiperiodic tori, see e.g. [3], [4], [6], [9]) in well-chosen spaces of observables, or they correspond to the asymptotic ergodic dynamics on (strange) attractors, therefore carrying no information on stability.

\section{A. Eigenfunctions of the Koopman operator}

The point spectrum of the Koopman operator is the set of values $\lambda \in \mathbb{C}$ such that

$$
U^{t} \phi_{\lambda}=e^{\lambda t} \phi_{\lambda}
$$

for a function $\phi_{\lambda} \in \mathcal{F} \neq 0$. The values $\lambda$ are the Koopman eigenvalues and the associated functions $\phi_{\lambda}$ are the Koopman eigenfunctions. If $F \in C^{1}(X)$, the eigenvalue equation can also be expressed, according to (3), as

$$
L_{U} \phi_{\lambda}=F \cdot \nabla \phi_{\lambda}=\lambda \phi_{\lambda} .
$$

It can be shown that if $\phi_{\lambda_{1}}$ and $\phi_{\lambda_{2}}$ are two eigenfunctions, then $\phi_{\lambda_{1}}^{k_{1}} \phi_{\lambda_{2}}^{k_{2}}$ is an eigenfunction associated with the eigenvalue $k_{1} \lambda_{1}+k_{2} \lambda_{2}$ (see e.g. [11]). Also, it is noticeable that the Koopman eigenfunctions can be smooth, while the Perron-Frobenius eigenfunctions admit a singularity on the attractor.

The eigenfunctions are directly related to the dynamics of the system. For example, consider an eigenfunction at $\lambda=0$. It follows from (8) that this eigenfunction is constant along the trajectories, so that its level sets partition the state space into invariant regions. If one considers the non-degenerate intersections of the level sets of $n-1$ such (independent) eigenfunctions, we obtain a family of one-dimensional sets that correspond to the orbits of the system.

Remark 2: The spectral operator-theoretic framework presented in this paper yields an efficient method to compute all the orbits of the system, as an alternative to numerical integration. For instance, $C^{1}$ eigenfunctions $\phi_{\lambda_{i}}$, with $i=1, \ldots, n$, can be computed with the numerical method presented in Section $\mathrm{V}$ (when $A$ is a fixed point). Independent eigenfunctions associated with the eigenvalue 0 are obtained through the products $\phi_{\lambda_{i}}^{k_{i}} \phi_{\lambda_{j}}^{k_{j}}$, with $k_{i}=\lambda_{j}$ and $k_{j}=-\lambda_{i}$. The intersection of their level sets are the orbits of the system.

\section{B. Koopman eigenfunctions and stability}

The stability of the system is now investigated through the Koopman eigenfunctions. In Section III, we showed that the stability of the system is only related to the restriction $U_{A_{c}}^{t}$. Similarly, the following properties show that only a subset of the eigenfunctions is related to the stability property.

Property 1: If $\phi_{\lambda} \in \mathcal{F}_{A_{c}}$ is an eigenfunction of $U^{t}$, then $\phi_{\lambda}$ is also an eigenfunction of the restriction $U_{A_{c}}^{t}$, associated with the same eigenvalue. Moreover, if $\phi_{\lambda} \in C^{0}(X)$ and if the attractor $A$ is globally stable, then $\Re\{\lambda\}<0$.

Proof: The first part is trivial, since $A_{c}$ is invariant under $U^{t}$. The second part follows from Proposition 1 .

Property 2: If $\phi_{\lambda} \notin \mathcal{F}_{A_{c}}$ is an eigenfunction of $U^{t}$, then the restriction $\left.\phi_{\lambda}\right|_{A}$ of $\phi_{\lambda}$ to $A$ is an eigenfunction of $U_{A}^{t}$, associated with the same eigenvalue. Moreover, if $\phi_{\lambda} \in C^{0}(X)$, then $\Re\{\lambda\}=0$.

\section{Proof: One has}

$$
U_{A}^{t}\left(\left.\phi_{\lambda}\right|_{A}\right)=\left.\left(U^{t} \phi_{\lambda}\right)\right|_{A}=\left.e^{\lambda t} \phi_{\lambda}\right|_{A} .
$$

In addition, suppose that $\Re\{\lambda\} \neq 0$. If $\Re\{\lambda\}>0$, then the eigenfunction is not bounded on $A$. If $\Re\{\lambda\}<0$, one has $\lim _{t \rightarrow \infty} \phi_{\lambda}\left(\varphi^{t}(x)\right)=0 \forall x$. In this case, consider a point $x_{\omega} \in A$ which belongs to the limit set of $\varphi^{t}(x)$. There exists a sequence $t_{n}$ such that $t_{n} \rightarrow \infty$ and $\varphi^{t_{n}}(x) \rightarrow x_{\omega}$ as $n \rightarrow \infty$. Then, it follows from the continuity of $\phi_{\lambda}$ that

$$
\phi_{\lambda}\left(x_{\omega}\right)=\phi_{\lambda}\left(\lim _{n \rightarrow \infty} \varphi^{t_{n}}(x)\right)=\lim _{n \rightarrow \infty} \phi_{\lambda}\left(\varphi^{t_{n}}(x)\right)=0 .
$$

In both cases, there is a contradiction with the fact that $\phi_{\lambda}$ is continuous and has nonzero value for some points of the attractor, which concludes the proof. 
The properties imply that the eigenfunctions relevant for the stability analysis - i.e. related to $U_{A_{c}}^{t}$ - lie in $\mathcal{F}_{A_{c}}$ and are characterized by $\Re\{\lambda\}<0$. The other eigenfunctions, associated with purely imaginary eigenvalues, provide no information on stability but are related to asymptotic properties of the trajectories on the attractor. For instance, they can be used to compute periodic invariant sets on the attractor [10] or to compute the so-called isochrons of (quasi)periodic attractors [8].

The following proposition provides a general result drawing the link between the eigenfunctions in $\mathcal{F}_{A_{c}}$ and the global stability of the system.

Proposition 3: Suppose that the Koopman operator admits the eigenfunctions $\phi_{\lambda_{i}} \in C^{0}(X)$ with the eigenvalues $\Re\left\{\lambda_{i}\right\}<0, i=1, \ldots, m$. Then, the set

$$
M=\bigcap_{i=1}^{m}\left\{x \in X \mid \phi_{\lambda_{i}}(x)=0\right\} \supseteq A
$$

is globally asymptotically stable.

Proof: Since $\Re\left\{\lambda_{i}\right\}<0$, the equality (8) implies that $\lim _{t \rightarrow \infty} \phi_{\lambda_{i}}\left(\varphi^{t}(x)\right)=0 \forall x$. Since $\phi_{\lambda_{i}}$ is continuous, (10) holds so that the limit set of every trajectory is contained in the zero level set of $\phi_{\lambda_{i}}$. This concludes the proof.

The proof is inspired from the proof of the KrasovskiiLaSalle principle (see e.g. [5]). However, the differentiability of the eigenfunctions is not required here since we know $a$ priori that these eigenfunctions are decreasing (and asymptotically converge to zero) along the trajectories.

Remark 3: If $A$ is an invariant set, but not an attractor, the set corresponding to the intersections of the zero level sets of eigenfunctions $\phi_{\lambda_{i}} \in \mathcal{F}_{A_{c}}$, with $\Re\left\{\lambda_{i}\right\} \geq 0$, is the stable set of $A$.

\section{The case of a fixed point}

In this section, we develop the framework for the particular case of a stable fixed point $x^{*} \in X$. We assume that the vector field $F \in C^{1}(X)$ and that the Jacobian matrix $\mathbf{J}$ of $F$ at $x^{*}$ has $n$ eigenvalues $\lambda_{i}^{*}$ with $\Re\left\{\lambda_{i}^{*}\right\}<0$. We also suppose that the corresponding eigenvectors $v_{i}$ are independent.

Proposition 4: Assume that $X$ is a connected set. If, for all $\lambda_{i}^{*}$, the Koopman operator admits an eigenfunction $\phi_{\lambda_{i}^{*}} \in$ $C^{1}(X)$, with $\nabla \phi_{\lambda_{i}^{*}}\left(x^{*}\right) \neq 0$, then $x^{*}$ is globally stable in $X$.

Proof: Consider the first order Taylor approximations

$$
\phi_{\lambda_{i}^{*}}(x)=\nabla \phi_{\lambda_{i}^{*}}\left(x^{*}\right)\left(x-x^{*}\right)+o\left(\left\|x-x^{*}\right\|\right)
$$

(where we used the relationship $\phi_{\lambda_{i}^{*}}\left(x^{*}\right)=0$ that rigorously follows from the contraposition of Property 2) and

$$
F(x)=\mathbf{J}\left(x-x^{*}\right)+o\left(\left\|x-x^{*}\right\|\right) .
$$

Injecting these two approximations into (8) yields

$$
\mathbf{J}^{T} \nabla \phi_{\lambda_{i}^{*}}\left(x^{*}\right)=\lambda_{i}^{*} \nabla \phi_{\lambda_{i}^{*}}\left(x^{*}\right),
$$

so that $\nabla \phi_{\lambda_{i}^{*}}\left(x^{*}\right)$ is equal (up to a multiplicative constant) to the left eigenvector $\tilde{v}_{i}$ of $\mathbf{J}$. In particular, $\Re\left\{\nabla \phi_{\lambda_{i}^{*}}\left(x^{*}\right)\right\}$ and $\Im\left\{\nabla \phi_{\lambda_{i}^{*}}\left(x^{*}\right)\right\}$ (when $\lambda_{i}^{*}$ is complex) are respectively parallel to $\Re\left\{\tilde{v}_{i}\right\}$ and $\Im\left\{\tilde{v}_{i}\right\}$. In a small neighborhood $V$ of $x^{*}$, the zero level sets of $\Re\left\{\phi_{\lambda_{i}^{*}}\right\}$ and $\Im\left\{\phi_{\lambda_{i}^{*}}\right\}$ are tangent to a hyperplane whose normal is $\Re\left\{\tilde{v}_{i}\right\}$ and $\Im\left\{\tilde{v}_{i}\right\}$, respectively. Since the $n$ (non-zero) eigenvectors $\Re\left\{\tilde{v}_{i}\right\}$ and $\Im\left\{\tilde{v}_{i}\right\}$ are independent, the intersection in $V$ of the zero level sets of the $n$ different functions $\Re\left\{\phi_{\lambda_{i}^{*}}\right\}$ and $\Im\left\{\phi_{\lambda_{i}^{*}}\right\}$-or equivalently of the eigenfunctions $\phi_{\lambda_{i}^{*}}$ - can only be $x^{*}$.

Next, we show that the zero level sets cannot have another intersection in $X$. Suppose that there is another intersection. This defines another invariant set which is not connected to the fixed point. Therefore, the boundary $\delta \Omega$ of the basin of attraction $\Omega$ of $x^{*}$ has a non empty intersection with $X$, since $X$ is a connected set. Moreover, since $X$ is forward invariant, $\delta \Omega \cap X$ is also forward invariant and contains the limit sets of its trajectories. Consider a point $x_{\omega} \in \delta \Omega \cap X$ that belongs to a limit set. By definition and continuity of the eigenfunctions, we have $\phi_{\lambda_{i}^{*}}\left(x_{\omega}\right)=0 \forall i$ (see (10)). Also, for any arbitrarily small neighborhood $V_{\epsilon}$ of $x_{\omega}$ and for all $x_{\epsilon} \in V_{\epsilon} \cap \Omega$, there exist a point $x_{V} \in V$ with $x_{V} \neq x^{*}$ and a constant $T>0$ such that $\varphi^{-T}\left(x_{V}\right)=x_{\epsilon}$. There is at least one eigenfunction that satisfies $\left|\phi_{\lambda_{i}^{*}}\left(x_{V}\right)\right|=C>0$, or equivalently $\left|\phi_{\lambda_{i}^{*}}\left(x_{\epsilon}\right)\right|=C \exp \left(-\Re\left\{\lambda_{i}^{*}\right\} T\right)>0$. Therefore, $\phi_{\lambda_{i}^{*}}$ is not continuous in $V_{\epsilon} \subset X$, which is a contradiction. Finally, the result follows from Proposition 3, with $M=$ $\left\{x^{*}\right\}$.

This result can be seen as the global equivalent of the well-known local stability result for a fixed point. While local stability is implied by stable eigenvalues of $\mathbf{J}$, global stability is implied by $C^{1}$ eigenfunctions of $U^{t}$ (with the same eigenvalues). Also, global stability relies more on the continuous differentiability of the eigenfunctions than on the existence of the eigenfunctions themselves.

1) Lyapunov functions and contracting metrics: (See also [9].) Continuously differentiable Koopman eigenfunctions are intimately related to Lyapunov functions and contracting metrics. Under the assumption of Proposition 4, the eigenfunctions yield for instance the special Lyapunov functions

$$
\mathcal{V}(x)=\left(\sum_{i=1}^{n}\left|\phi_{\lambda_{i}^{*}}(x)\right|^{p}\right)^{1 / p}
$$

with the integer $p \geq 1$. According to (8), they satisfy $\mathcal{V}\left(\varphi^{t}(x)\right) \leq \exp \left(\Re\left\{\lambda_{1}^{*}\right\} t\right) \mathcal{V}(x)$ for $x \in X$, where $\lambda_{1}^{*}$ is the eigenvalue closest to the imaginary axis. Note that these Lyapunov functions are not necessarily smooth. In addition, the eigenfunctions define the metrics

$$
\mathcal{M}(x, y)=\left(\sum_{i=1}^{n}\left|\phi_{\lambda_{i}^{*}}(x)-\phi_{\lambda_{i}^{*}}(y)\right|\right)^{1 / p}
$$

which are exponentially contracting on $X$, since $\mathcal{M}\left(\varphi^{t}(x), \varphi^{t}(y)\right) \leq \exp \left(\Re\left\{\lambda_{1}^{*}\right\} t\right) \mathcal{M}(x, y)$. These metrics are non-quadratic and could be considered through the differential framework recently developed in [2].

2) Other properties of the eigenfunctions: In contrast with a Lyapunov function, the Koopman eigenfunctions provide additional information than mere stability. For instance, they define new coordinates $z_{i}=\phi_{\lambda_{i}^{*}}(x)$ associated with linear 
dynamics $\dot{z}_{i}=\lambda_{i}^{*} z_{i}$ [6] and are therefore related to the rate of convergence of the trajectories. In particular, the level sets of the eigenfunction $\phi_{\lambda_{1}^{*}}$ correspond to the notion of isostables defined in [9]: they are the sets of points that converge "synchronously" toward the fixed point.

\section{NUMERICAL COMPUTATION}

In the case of fixed points, Koopman eigenfunctions can be computed through Laplace averages evaluated along the trajectories of the system [9], [11]. Here, we propose a numerical method for computing smooth Koopman eigenfunctions, which does not require the integration of trajectories. Exploiting the result of Proposition 4, this method can be used in a systematic way to establish global stability on a given subset of the state space or to estimate the basin of attraction of the fixed point.

\section{A. The method}

When the vector field $F$ is analytic (and provided that the eigenvalues $\lambda_{i}^{*}$ are nonresonant), the Koopman eigenfunctions are also analytic (at least in a neighborhood of the fixed point), as a consequence of the Poincaré linearization theorem (see e.g. [3]). In this case, it is natural to consider the Taylor decomposition

$\phi_{\lambda}(x)=\sum_{k_{1}=0}^{\infty} \cdots \sum_{k_{n}=0}^{\infty} \phi_{\lambda}^{\left(k_{1}, \ldots, k_{n}\right)}\left(x_{1}-x_{1}^{*}\right)^{k_{1}} \cdots\left(x_{n}-x_{n}^{*}\right)^{k_{n}}$

with

$$
\phi_{\lambda}^{\left(k_{1}, \ldots, k_{n}\right)}=\left.\frac{1}{k_{1} ! \cdots k_{n} !} \frac{\partial^{k_{1}+\cdots+k_{n}} \phi_{\lambda}}{\partial x_{1}^{k_{1}} \cdots \partial x_{n}^{k_{n}}}\right|_{x^{*}}
$$

and with $x=\left(x_{1}, \ldots, x_{n}\right)$. Note that this polynomial approximation resembles the sum of squares (SOS) method used for the computation of Lyapunov functions [12].

One can also compute a Taylor expansion for $F(x)=$ $\left(F_{1}(x), \ldots, F_{n}(x)\right)$, with the coefficients

$$
F_{l}^{\left(k_{1}, \ldots, k_{n}\right)}=\left.\frac{1}{k_{1} ! \cdots k_{n} !} \frac{\partial^{k_{1}+\cdots+k_{n}} F_{l}}{\partial x_{1}^{k_{1}} \cdots \partial x_{n}^{k_{n}}}\right|_{x^{*}} .
$$

Then, by injecting the Taylor expansions of $\phi_{\lambda}$ and $F$ into the eigenvalue equation (9) and by identifying the terms in $\left(x_{1}-x_{1}^{*}\right)^{k_{1}} \cdots\left(x_{n}-x_{n}^{*}\right)^{k_{n}}$, we obtain the relationship

$$
\begin{aligned}
& \sum_{j_{1}=0}^{k_{1}} \cdots \sum_{j_{n}=0}^{k_{n}} \sum_{l=1}^{n}\left(k_{l}-j_{l}+1\right) F_{l}^{\left(j_{1}, \cdots, j_{n}\right)} \\
& \quad \times \phi_{\lambda}^{\left(k_{1}-j_{1}, \ldots, k_{l-1}-j_{l-1}, k_{l}-j_{l}+1, k_{l+1}-j_{l+1}, \ldots, k_{n}-j_{n}\right)} \\
& =\lambda \phi_{\lambda}^{\left(k_{1}, \ldots, k_{n}\right)} \quad \forall\left(k_{1}, \ldots, k_{n}\right) \in \mathbb{N}^{n} .
\end{aligned}
$$

For each $s \geq 1 \in \mathbb{N}$, consider a vector $\Phi_{\lambda}^{(s)}$ whose components are the coefficients $\phi_{\lambda}^{\left(k_{1}, \cdots, k_{n}\right)}$, with $\sum_{j} k_{j}=s$. In the case $s=1$, (12) is rewritten as

$$
\mathbf{J}^{T} \Phi_{\lambda}^{(1)}=\lambda \Phi_{\lambda}^{(1)}
$$

and we recover the equality (11). This implies that $\lambda$ must be one of the eigenvalues $\lambda_{i}^{*}$ of $\mathbf{J}$ and that $\Phi_{\lambda_{i}^{*}}^{(1)}=\nabla \phi_{\lambda_{i}^{*}}\left(x^{*}\right)$ is the corresponding left eigenvector $\tilde{v}_{i}$.
For $s>1$, (12) is expressed as

$$
\mathbf{H}^{(s)} \Phi_{\lambda_{i}^{*}}^{(s)}=\lambda_{i}^{*} \Phi_{\lambda_{i}^{*}}^{(s)}+\mathbf{G}^{(s)}\left(\Phi_{\lambda_{i}^{*}}^{(1)}, \ldots, \Phi_{\lambda_{i}^{*}}^{(s-1)}\right)
$$

where $\mathbf{H}^{(s)}$ is a matrix and $\mathbf{G}^{(s)}$ is a (vector-valued) linear function. All the coefficients can be computed by solving (13), for the successive $s$ values. (Note that (13) resembles the method of Carleman embedding [1].)

It is important to note that the above method could be adapted to study the stability of other types of attractors. For instance, the case of limit cycles could be considered by combining a Taylor expansion in the direction transversal to the attractor with a Fourier expansion in the direction tangential to the attractor. The only caveat is that an a priori knowledge of the attractor is required in that case. Otherwise, the eigenfunctions of the Koopman operator might also be obtained-without integration of the trajectories-through other methods similar to those developed in [15] (e.g. discretization method, convex linear programming methods).

\section{B. Examples}

1) Example 1: To illustrate the numerical method, we first consider the dynamics

$$
\begin{aligned}
& \dot{x}_{1}=-x_{1}-x_{1}^{2} x_{2}-x_{2}^{3} . \\
& \dot{x}_{2}=-x_{2}+x_{1} x_{2}^{2}+x_{1}^{3} .
\end{aligned}
$$

In polar coordinates, the dynamics are $\dot{r}=-r, \dot{\theta}=r^{2}$, so that the origin is globally stable in $\mathbb{R}^{2}$. This is coherent with the fact that our numerical method shows that there exist two (independent) Koopman eigenfunctions, both associated with the eigenvalue $\lambda_{1}^{*}=\lambda_{2}^{*}=-1$ (Fig. 1).
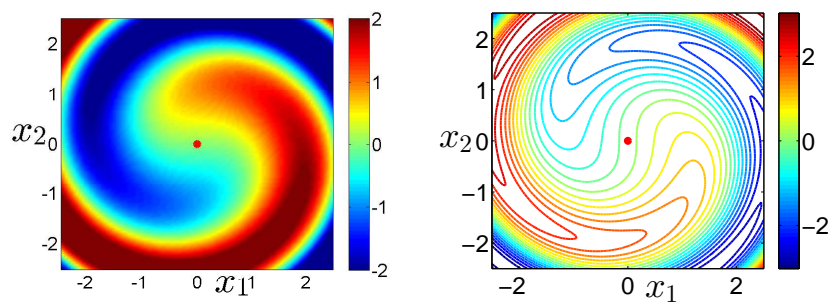

Fig. 1. In Example 1, the existence of smooth eigenfunctions implies global stability of the fixed point (here in a square $[-2.5,2.5] \times[-2.5,2.5])$. Left. Color plot of the eigenfunction $\phi_{\lambda_{1}^{*}}$. Right. Level sets of the eigenfunction $\phi_{\lambda_{2}^{*}}$. (The eigenfunctions are computed with a Taylor expansion to the 40th order.)

\section{2) Example 2: The Van der Pol dynamics}

$$
\begin{aligned}
& \dot{x}_{1}=-x_{2} \\
& \dot{x}_{2}=x_{1}-x_{2}+x_{1}^{2} x_{2}
\end{aligned}
$$

admit a stable fixed point at the origin, with a basin of attraction delimited by an unstable limit cycle. The two (complex) eigenfunctions computed through the method tend to infinity as they come close to the limit cycle. They therefore provide an accurate evaluation of the basin of attraction of the fixed point (Fig. 2). 


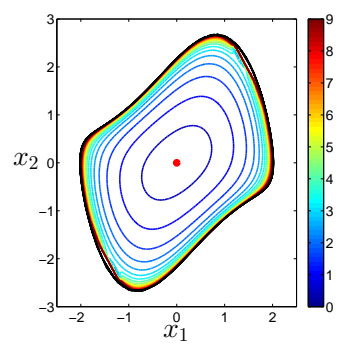

Fig. 2. For the Van der Pol dynamics, the level sets of $\left|\phi_{\lambda_{1}}^{*}\right|$ concentrate near the unstable limit cycle (black curve). The basin of attraction of the fixed point is therefore well-approximated by the method. (The eigenfunctions are computed with a Taylor expansion to the 100th order.)

3) Example 3: Consider the dynamics (slightly modified from [14])

$$
\begin{aligned}
& \dot{x}_{1}=-2 x_{1}+x_{1}^{2}-x_{2}^{2} . \\
& \dot{x}_{2}=-2.5 x_{2}+2 x_{1} x_{2} .
\end{aligned}
$$

While the origin is globally stable on $\mathbb{R}^{2} \backslash\left\{\left(x_{1}, x_{2}\right) \mid x_{1} \geq\right.$ $\left.2, x_{2}=0\right\}$, our numerical method shows the existence of analytic eigenfunctions only on a disk of radius 2 , and therefore proves global stability only on that region (Fig. 3). This is actually in accordance with the Poincare linearization theorem, which implies that the eigenfunctions are analytical on the larger disk (centered at the stable fixed point) that does not contain another fixed point. In this present case, there is precisely an unstable fixed point that creates a singularity at $(2,0)$. Note that the eigenfunctions can still be obtained in the entire basin of attraction through the computation of Laplace averages [11].
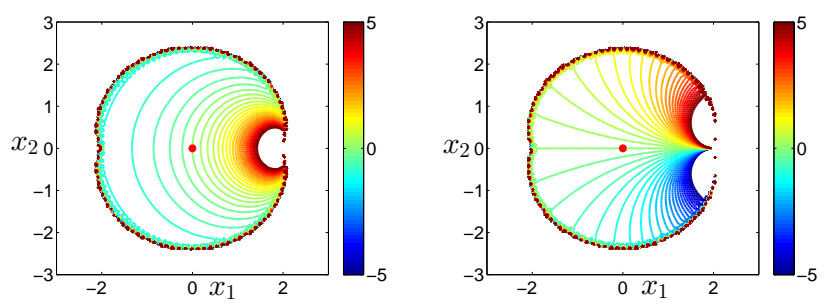

Fig. 3. For the dynamics of Example 3, the numerical method implies global stability on a disk of radius 2. Outside this disk, the Koopman eigenfunctions are not analytic and admit a singularity at the unstable fixed point $(2,0)$. Left. Level sets of the eigenfunction $\phi_{\lambda_{1}^{*}}$ with $\lambda_{1}^{*}=-2$. Right. Level sets of the eigenfunction $\phi_{\lambda_{2}^{*}}$ with $\lambda_{2}^{*}=-2.5$.(The eigenfunctions are computed with a Taylor expansion to the 100th order.)

\section{CONCLUSION}

We have developed a new approach to global stability analysis, based on the spectral properties of the Koopman operator. We have shown that a specific restriction of this operator captures the stability properties of the attractor. More importantly, a strong connection has been established between global stability and the existence of continuously differentiable Koopman eigenfunctions. The results are closely related to the notions of Lyapunov function and contracting metrics, but also pave the way for an alternative to classical stability theory.

In the case of a stable fixed point, a method is proposed to compute smooth Koopman eigenfunctions. This method is based on Taylor approximations and does not require the computation of the system trajectories. It yields an efficient numerical scheme that can be used to investigate the global stability properties of the fixed point (e.g. size of the basin of attraction).

A key point of the operator-theoretic framework is that it is general enough to encompass a broad class of attractors. Therefore, we envision that our results could be extended to stable limit cycles and possibly to more complex attractors (e.g. strange attractors). However, other numerical methods-which still need to be developed-might be required. Another promising direction is to adapt the framework to the stability analysis of non-autonomous systems and input-output systems.

\section{ACKNOWLEDGMENTS}

The work was funded by Army Research Office Grant W911NF-11-1-0511, with Program Manager Dr. Sam Stanton.

\section{REFERENCES}

[1] T. Carleman. Application de la théorie des équations integrales lineaires aux systèmes d'équations différentielles nonlinéaires. Acta Mathematica, 59:63-68, 1932.

[2] F. Forni and R. Sepulchre. A differential Lyapunov framework for contraction analysis. http://arxiv.org/abs/1208.2943.

[3] P. Gaspard, G. Nicolis, A. Provata, and S. Tasaki. Spectral signature of the pitchfork bifurcation: Liouville equation approach. Physical Review E, 51(1):74, 1995.

[4] P. Gaspard and S. Tasaki. Liouvillian dynamics of the hopf bifurcation. Physical Review E, 64(5):056232, 2001.

[5] H. K. Khalil and J. W. Grizzle. Nonlinear systems, volume 3. Prentice hall New Jersey, 1996.

[6] Y. Lan and I. Mezić. Linearization in the large of nonlinear systems and Koopman operator spectrum. Physica D, 242:42-53, 2013.

[7] A. Lasota and M. C. Mackey. Chaos, Fractals, and Noise: stochastic aspects of dynamics. Springler-Verlag, 1994.

[8] A. Mauroy and I. Mezić. On the use of Fourier averages to compute the global isochrons of (quasi)periodic dynamics. Chaos, 22(3):033112, 2012.

[9] A. Mauroy, I. Mezić, and J. Moehlis. Isostables, isochrons, and Koopman spectrum for the action-angle representation of stable fixed point dynamics. Physica D, 261:19-30, October 2013.

[10] I. Mezić. Spectral properties of dynamical systems, model reduction and decompositions. Nonlinear Dynamics, 41(1-3):309-325, 2005.

[11] I. Mezić. Analysis of fluid flows via spectral properties of Koopman operator. Annual Review of Fluid Mechanics, 45, January 2013.

[12] P. A. Parrilo. Structured semidefinite programs and semialgebraic geometry methods in robustness and optimization. $\mathrm{PhD}$ thesis, California Institute of Technology, 2000.

[13] R. Rajaram, U. Vaidya, M. Fardad, and B. Ganapathysubramanian. Stability in the almost everywhere sense: A linear transfer operator approach. Journal of Mathematical Analysis and Applications, 368(1):144-156, 2010.

[14] A. Rantzer. A dual to Lyapunov's stability theorem. Systems \& Control Letters, 42(3):161-168, 2001.

[15] U. Vaidya and P. G. Mehta. Lyapunov measure for almost everywhere stability. IEEE Transactions on Automatic Control, 53(1):307-323, 2008. 